\title{
The Thousand-Pulsar-Array programme on MeerKAT I: Science objectives and first results
}

\author{
Simon Johnston $^{1 \star}$, A. Karastergiou ${ }^{2,3,4}$, M. J. Keith ${ }^{5}$, X. Song ${ }^{5}$, P. Weltevrede ${ }^{5}$, \\ F. Abbate ${ }^{6,7}$, M. Bailes ${ }^{8,9}$, S. Buchner ${ }^{10}$, F. Camilo ${ }^{10}$, M. Geyer ${ }^{10}$, B. Hugo ${ }^{10,4}$, A. Jameson ${ }^{8,9}$, \\ M. Kramer ${ }^{1,5}$, A. Parthasarathy ${ }^{8,9}$, D. J. Reardon ${ }^{8,9}$, A. Ridolfi ${ }^{7,11}$, M. Serylak ${ }^{10,3}$, \\ R. M. Shannon ${ }^{8,9}$, R. Spiewak ${ }^{8,9}$, W. van Straten $^{12}$, V. Venkatraman Krishnan ${ }^{11}$, F. Jankowski ${ }^{5}$, \\ B. W. Meyers ${ }^{13,14}$, L. Oswald ${ }^{2}$, B. Posselt ${ }^{2,15}$, C. Sobey ${ }^{16}$, A. Szary ${ }^{17,18}$, J. van Leeuwen ${ }^{17,19}$ \\ ${ }^{1}$ CSIRO Astronomy and Space Science, Australia Telescope National Facility, PO Box 76, Epping NSW 1710, Australia \\ ${ }^{2}$ Oxford Astrophysics, Denys Wilkinson Building, Keble Road, Oxford, OX1 3RH, UK. \\ ${ }^{3}$ Physics Department, University of the Western Cape, Cape Town 7535, South Africa \\ ${ }^{4}$ Department of Physics and Electronics, Rhodes University, PO Box 94, Grahamstown 6140, South Africa \\ 5 Jodrell Bank Centre for Astrophysics, The University of Manchester, Alan Turing Building, Manchester, M13 9PL, United Kingdom \\ ${ }^{6}$ Dipartimento di Fisica 'G. Occhialini', Universita 'degli Studi Milano Bicocca, Piazza della Scienza 3, I-20126 Milano, Italy \\ ${ }^{7}$ INAF - Osservatorio Astronomico di Cagliari, Via della Scienza 5, I-09047 Selargius (CA), Italy \\ ${ }^{8}$ Centre for Astrophysics and Supercomputing, Swinburne University of Technology, PO Box 218, Hawthorn, VIC 3122, Australia \\ ${ }^{9}$ ARC Centre of Excellence for Gravitational Wave Discovery (OzGrav) \\ ${ }^{10}$ South African Radio Astronomy Observatory (SARAO), 2 Fir Street, Black River Park, Observatory, Cape Town, 7925 \\ ${ }^{11}$ Max-Planck-Institut fuer Radioastronomie, Auf dem Huegel 69, D-53121 Bonn, Germany. \\ ${ }^{12}$ Institute for Radio Astronomy \& Space Research, Auckland University of Technology, Private Bag 92006, Auckland 1142, New Zealand \\ ${ }^{13}$ Dept. of Physics and Astronomy, University of British Columbia, 6224 Agricultural Road, Vancouver, B.C., V6T 1Z1, Canada \\ ${ }^{14}$ International Centre for Radio Astronomy Research, Curtin University, Bentley, WA 6102, Australia \\ ${ }^{15}$ Department of Astronomy and Astrophysics, The Pennsylvania State University, 525 Davey Laboratory, University Park, PA 16802, USA \\ ${ }^{16}$ CSIRO Astronomy and Space Science, PO Box 1130, Bentley, WA 6102, Australia \\ ${ }^{17}$ ASTRON, Netherlands Institute for Radio Astronomy, Oude Hoogeveensedijk 4, 7991 PD, Dwingeloo, The Netherlands \\ ${ }^{18}$ Janusz Gil Institute of Astronomy, University of Zielona Góra, Lubuska 2, 65-265 Zielona Góra, Poland \\ ${ }^{19}$ Anton Pannekoek Institute for Astronomy, University of Amsterdam, Science Park 904, 1098 XH Amsterdam, Netherlands
}

Last updated; in original form

\begin{abstract}
We report here on initial results from the Thousand Pulsar Array (TPA) programme, part of the Large Survey Project "MeerTime" on the MeerKAT telescope. The interferometer is used in tied-array mode in the band from 856 to $1712 \mathrm{MHz}$, and the wide band coupled with the large collecting area and low receiver temperature make it an excellent telescope for the study of radio pulsars. The TPA is a 5 year project which aims to observe (a) more than 1000 pulsars to obtain high-fidelity pulse profiles, (b) some 500 of these pulsars over multiple epochs, (c) long sequences of single-pulse trains from several hundred pulsars. The scientific outcomes from the programme will include determination of pulsar geometries, the location of the radio emission within the pulsar magnetosphere, the connection between the magnetosphere and the crust and core of the star, tighter constraints on the nature of the radio emission itself as well as interstellar medium studies. First results presented here include updated dispersion measures, 26 pulsars with Faraday rotation measures derived for the first time and a description of interesting emission phenomena observed thus far.
\end{abstract}

Key words: pulsars:general, instrumentation:interferometers

\section{INTRODUCTION}

^ E-mail: simon.johnston@csiro.au

Observations of radio pulsars have been a key plank of radio astronomy since their discovery in the late 1960s (Hewish et al. 1968). Over the past few decades, pulsar observations have gen- 
erally been the preserve of the world's largest single dishes, including the Parkes, Effelsberg, Arecibo, Lovell and Green Bank Telescopes. More recently, the power of interferometers has been harnessed towards pulsar observing including the Giant Metrewave Radio Telescope (GMRT) and the LOw Frequency ARray (LOFAR). The Parkes radio telescope has for decades been the most sensitive single dish in the southern hemisphere and has a long and glorious history in pulsar astronomy from the detection of the first pulsar glitch (Radhakrishnan \& Manchester 1969), through to the discovery of the double pulsar (Burgay et al. 2003; Lyne et al. 2004), Fast Radio Bursts (Lorimer et al. 2007; Thornton et al. 2013) and beyond. The commissioning of the MeerKAT telescope provides an alternative for observations of pulsars in the southern skies.

MeerKAT is an interferometer located in the Karoo, in South Africa's Northern Cape Province (Jonas \& MeerKAT Team 2016). In its current configuration it consists of 64 unblocked aperture dishes, each with an effective diameter of $13.97 \mathrm{~m}$. The 64 antennas in the array can be coherently phased together to form a highly sensitive 'tied-array beam' which is used for pulsar observations. The L-band receiver has a band which extends from 856 to $1712 \mathrm{MHz}$, and has a receiver temperature of $18 \mathrm{~K}$. The overall system equivalent flux density is $7.5 \mathrm{Jy}$, this compares to the Parkes value of $35 \mathrm{Jy}$ in the same frequency band (Hobbs et al. 2019).

MeerTime is an approved Large Survey Project on the MeerKAT telescope for observing known radio pulsars (Bailes et al. 2019). Pulsars can be used as a tool for cosmological tests of the stochastic gravitational wave background (e.g. Shannon et al. 2015; Arzoumanian et al. 2018), tests of theories of gravity (e.g. Kramer et al. 2006b; Freire et al. 2012), and understanding the equation of state of nuclear matter (e.g. Antoniadis et al. 2013; Özel \& Freire 2016). In addition observations of pulsars give insight into the internal structure of neutron stars (e.g. Antonopoulou et al. 2018), the workings of the pulsar magnetosphere (e.g. Dyks \& Rudak 2015; Ilie et al. 2019), and the structure of the Galaxy (e.g. Noutsos et al. 2008; Yao et al. 2017).

In the sections below we first outline the science case for the Thousand-Pulsar-Array programme within the MeerTime project. We then describe the initial observations and system configuration. We demonstrate that the wide band of the MeerKAT receivers are ideal for measurements of dispersion measure and rotation measure and show examples of the high signal-to-noise ratio observations on individual pulsars. We end by briefly discussing the future of the project.

\section{THE THOUSAND-PULSAR-ARRAY PROGRAMME}

The Thousand-Pulsar-Array (TPA) programme is part of MeerTime, the main project for observations of known pulsars on the MeerKAT telescope (Bailes et al. 2019). The selection criteria for the TPA is that the pulsars (a) are south of declination $+20^{\circ}$ (b) have positional errors less than $2^{\prime \prime}$ and (c) are not recycled or millisecond pulsars. There are three main observational aims of the TPA. The first is to obtain a homogeneous set of polarimetric profiles for more than 1000 pulsars with sufficient signal-to-noise ratio to measure the standard parameters such as dispersion measure (DM) and rotation measure (RM), pulse widths and polarization properties. The second is to obtain observations with a regular cadence over a total time window of 5 years of at least 500 pulsars with sufficient observing time per observation to enable detection of pulse profile changes at the $10 \%$ level. The third is to obtain sequences of single pulse trains in full polarization for more than 500 pulsars with sufficient signal-to-noise ratio to allow standard single-pulse analysis. Finally, the TPA schedule needs to be flexible to respond to glitches, state changes and other events which require follow-up observations. We estimate the total observing time to be $\sim 750 \mathrm{hr}$ spread over 5 years, with the bulk of the time allocated to the regular monitoring project.

The scientific goals that motivate these observations include the determination of the geometry for each pulsar, the location of the radio emission within the pulsar magnetosphere, the connection between the magnetosphere and the crust/core of the star, and the nature of the radio emission itself. In addition much can be gleaned of the properties of the interstellar medium (ISM) from the data. Each of these topics has a body of literature behind it; here we briefly summarise some open questions and a selection of recent references.

Fundamental to understanding individual pulsars, their properties and the pulsar population as a whole is the geometry of these objects and how it evolves with time. Determining the geometry of a large sample of pulsars remains fraught with uncertainties (Rookyard et al. 2015) largely due to magnetospheric and relativistic distortions to the underlying simplicity of the rotating vector model (Radhakrishnan \& Cooke 1969). The evolution with time is a key theoretical question (Arzamasskiy et al. 2017) with somewhat conflicting observational results (Lyne et al. 2013; Johnston \& Karastergiou 2017).

Radio emission arises within the pulsar magnetosphere, but the question regarding emission heights and the general 3D structure of the pulsar radio beam remains open (e.g. Philippov et al. 2015). Conal structures (Rankin 1993; Olszanski et al. 2019), patchy structures (Lyne \& Manchester 1988) or a hybrid (Karastergiou \& Johnston 2007) have been considered. More recently, interesting ideas pertaining to fan beams have emerged from theory and observations (Dyks \& Rudak 2015; Desvignes et al. 2019; Oswald et al. 2019).

The dynamic nature of the pulsar magnetosphere and the keys to the radio emission process are best revealed through the properties of individual pulses (Olszanski et al. 2019; Dyks 2019). Observations of the drifting sub-pulse phenomenon (Basu et al. 2016) and the associated carousel model (McSweeney et al. 2019) along with interpretation of the bi-drifting phenomenon (Szary \& van Leeuwen 2017; Wright \& Weltevrede 2017) provide insights into the emission mechanism. Currently the southern hemisphere pulsars have not been well searched or studied for sub-pulse drifting and other phenomena.

Evidence has emerged that there is a complex connection between the magnetosphere and the crust and core of a neutron star (Palfreyman et al. 2018). The ubiquity of, and link between, timing noise (Shannon \& Cordes 2010; Parthasarathy et al. 2019) and glitches (Fuentes et al. 2017; Haskell et al. 2018) poses many open questions. In addition, mode-switching involves changes in the profile and the spin-down torque of some pulsars (Kramer et al. 2006a; Lyne et al. 2010; Brook et al. 2016), the reasons for which remain unclear (Kerr et al. 2016; Stairs et al. 2019). Understanding the frequency dependence of pulsar emission, and monitoring the correlations between magnetospheric properties and rotation are key to the design of the TPA survey.

The frequency- and time-dependent ISM response function that filters the radio emission from pulsars poses several open questions. These can be broadly categorized into two groups, those pertaining to the characteristics of the ISM itself (Walker et al. 2008; Geyer et al. 2017; Kerr et al. 2018), and those that relate to how best to remove these effects from pulsar data to understand intrinsic pulsar properties (Walker et al. 2013; Pennucci 2019). The wide 
Table 1. Table showing DM measurements from MeerKAT observations that differ by more than $3 \mathrm{~cm}^{-3} \mathrm{pc}$ from the catalogue value.

\begin{tabular}{lrrrr} 
Pulsar & $\begin{array}{r}\text { Cat. DM } \\
\left(\mathrm{cm}^{-3} \mathrm{pc}\right)\end{array}$ & $\begin{array}{r}\text { uncert. } \\
\left(\mathrm{cm}^{-3} \mathrm{pc}\right)\end{array}$ & $\begin{array}{r}\text { Measured DM } \\
\left(\mathrm{cm}^{-3} \mathrm{pc}\right)\end{array}$ & $\begin{array}{r}\text { uncert. } \\
\left(\mathrm{cm}^{-3} \mathrm{pc}\right)\end{array}$ \\
\hline J0804-3647 & 196 & 5 & 186.8 & 0.2 \\
$\mathrm{~J} 0818-3049$ & 133.7 & 0.2 & 118.2 & 0.5 \\
J0902-6325 & 76 & 7 & 72.72 & 0.06 \\
J0905-4536 & 179.7 & & 196.2 & 0.3 \\
$\mathrm{~J} 0932-5327$ & 122 & 11 & 118.56 & 0.13 \\
$\mathrm{~J} 0952-3839$ & 167 & 3 & 162.88 & 0.11 \\
$\mathrm{~J} 1020-6026$ & 441.5 & 0.4 & 446.0 & 0.5 \\
$\mathrm{~J} 1231-4609$ & 76 & 7 & 67.4 & 0.3 \\
$\mathrm{~J} 1316-6232$ & 983.3 & 0.5 & 972.0 & 0.6 \\
$\mathrm{~J} 1427-4158$ & 71 & 3 & 65.4 & 0.2 \\
$\mathrm{~J} 1504-5621$ & 143 & 5 & 148.6 & 0.1 \\
$\mathrm{~J} 1525-5605$ & 338 & 3 & 333.5 & 0.4 \\
$\mathrm{~J} 1527-5552$ & 362.7 & 0.8 & 370.06 & 0.05 \\
$\mathrm{~J} 1536-3602$ & 96 & 6 & 85.7 & 0.4 \\
$\mathrm{~J} 1604-4718$ & 52.0 & 1.6 & 55.10 & 0.08 \\
$\mathrm{~J} 1651-7642$ & 80 & 10 & 59.9 & 0.9 \\
$\mathrm{~J} 1739+0612$ & 101.5 & 1.3 & 95.52 & 0.04 \\
$\mathrm{~J} 1759-2549$ & 431 & 5 & 424.0 & 0.2 \\
$\mathrm{~J} 1811-2439$ & 172.0 & 0.5 & 166.6 & 0.2 \\
$\mathrm{~J} 1814-1744$ & 792 & 1.6 & 820 & 2 \\
$\mathrm{~J} 1827-0750$ & 381 & 9 & 375.45 & 0.07 \\
$\mathrm{~J} 1832-0644$ & 578 & 7 & 574.3 & 0.3 \\
$\mathrm{~J} 1839-0402$ & 242 & 3 & 236.6 & 0.2 \\
$\mathrm{~J} 1839-0459$ & 243 & 3 & 237.0 & 0.3 \\
$\mathrm{~J} 1840-1207$ & 302.3 & 1.5 & 290.9 & 0.2 \\
$\mathrm{~J} 1842-0153$ & 434 & 5 & 427.20 & 0.06 \\
$\mathrm{~J} 1842-0415$ & 188 & 4 & 183.78 & 0.12 \\
$\mathrm{~J} 1844-0244$ & 429 & 3 & 425.3 & 0.2 \\
$\mathrm{~J} 1848-0023$ & 30.6 & 1 & 34.90 & 0.13 \\
$\mathrm{~J} 1849-0317$ & 42.9 & 2.8 & 39.55 & 0.06 \\
$\mathrm{~J} 1850-0006$ & 570 & 20 & 646 & 3
\end{tabular}

bandwidth of the TPA observations provides a large lever arm for measuring these effects.

Combining TPA and multiwavelength observations enables further understanding of pulsar physics. High-energy observations can, for example, provide independent constraints on the pulsar geometry, either through the analysis of their multiwavelength pulse profiles (Pierbattista et al. 2015; Giraud \& Pétri 2019) or pulsar wind nebulae (Ng \& Romani 2004; Klingler et al. 2018; Barkov et al. 2019). Multiwavelength monitoring of unusual pulsars such as the radio-loud magnetars and transition objects can shed light onto potential magnetospheric differences between the diverse neutron star populations (Camilo et al. 2018; Dai et al. 2018). Finally, the radio-X-ray correlation in mode-switching pulsars can put models of the pulsar magnetosphere to the test (Hermsen et al. 2013; Rigoselli et al. 2019).

The TPA is synergistic with related pulsar research being carried out world-wide. At the Parkes telescope, a sample of some 250 pulsars have been observed at $1.4 \mathrm{GHz}$ monthly since 2007 (Weltevrede et al. 2010; Johnston \& Kerr 2018). At the Lovell telescope, up to $40 \mathrm{yr}$ of timing has been done on a very large sample of pulsars with regular cadence (Espinoza et al. 2011). The UTMOST telescope, used as transit instrument since mid-2017, continues to monitor more than 300 pulsars at an observing frequency near $840 \mathrm{MHz}$ (Jankowski et al. 2019). Finally the CHIME telescope observes every pulsar in the northern sky at transit every day. Each of these programmes has its own strengths and weaknesses and will provide complementarity to the TPA programme.
Table 2. Rotation measures for pulsars without previous measurements.

\begin{tabular}{lrrr} 
Pulsar & $\begin{array}{r}\mathrm{RM} \\
\left(\mathrm{rad} \mathrm{m}^{-2}\right)\end{array}$ & $\begin{array}{r}\text { uncertainty } \\
\left(\mathrm{rad} \mathrm{m}^{-2}\right)\end{array}$ & $\begin{array}{r}\left\langle B_{\|}\right\rangle \\
(\mu \mathrm{G})\end{array}$ \\
\hline $\mathrm{J} 0630-0046$ & 44 & 2 & 0.55 \\
$\mathrm{~J} 1104-6103$ & -8.0 & 0.5 & -0.12 \\
$\mathrm{~J} 1130-6807$ & -150 & 6 & -1.24 \\
$\mathrm{~J} 1308-5844$ & 9.1 & 1.0 & 0.05 \\
$\mathrm{~J} 1434-6006$ & -361 & 3 & -1.34 \\
$\mathrm{~J} 1457-5902$ & 13.7 & 1.0 & 0.03 \\
$\mathrm{~J} 1502-5653$ & -253.7 & 0.7 & -1.61 \\
$\mathrm{~J} 1512-5431$ & 5.3 & 0.6 & 0.03 \\
$\mathrm{~J} 1514-5925$ & 6.4 & 1.0 & 0.04 \\
$\mathrm{~J} 1537-4912$ & 0.0 & 3 & 0.00 \\
$\mathrm{~J} 1604-4718$ & 1.6 & 1.1 & 0.03 \\
$\mathrm{~J} 1609-4616$ & -115.3 & 1.4 & 0.94 \\
$\mathrm{~J} 1634-5640$ & 159 & 5 & 1.32 \\
$\mathrm{~J} 1656-3621$ & -23.4 & 0.5 & -0.12 \\
$\mathrm{~J} 1716-4711$ & -221.8 & 1.2 & -0.96 \\
$\mathrm{~J} 1722-4400$ & -302.8 & 0.8 & -1.70 \\
$\mathrm{~J} 1726-4006$ & 284 & 4 & 1.26 \\
$\mathrm{~J} 1732-4156$ & -78 & 4 & -0.42 \\
$\mathrm{~J} 1758-1931$ & 295 & 3 & 1.76 \\
$\mathrm{~J} 1808-1020$ & 125.4 & 0.5 & 0.68 \\
$\mathrm{~J} 1808-1517$ & -169 & 4 & -1.01 \\
$\mathrm{~J} 1809-0743$ & -44 & 2 & -0.23 \\
$\mathrm{~J} 1811-2439$ & -82 & 2 & -0.59 \\
$\mathrm{~J} 1819-1008$ & 124 & 2 & 0.38 \\
$\mathrm{~J} 1820-0509$ & 77.6 & 0.8 & 0.92 \\
$\mathrm{~J} 1840-1207$ & -126.2 & 1.4 & -0.52
\end{tabular}

\section{OBSERVATIONS}

Observations for this programme commenced in 2019 February and as of the end of 2019 September a total of 315 pulsars have been observed. Generally the observations were carried out with at least 58 antennas used to form the tied-array beam. For the most part, data were recorded with 928 frequency channels between 896 and $1671 \mathrm{MHz}$ with 1024 time bins across the pulsar period for each of the Stokes parameters. The data are coherently de-dispersed at the known DM using DSPSR (van Straten \& Bailes 2011), folded at the topocentric spin-period of the pulsar using its ephemeris ${ }^{1}$ and written to disk every 8 seconds for the duration of the observation. In addition to the fold-mode data stream, a second stream is recorded at a sampling rate of $32 \mu$ s for each channel, necessary for the study of single pulses. The data are written to disk in PSRFITs format for data reduction with the PSRCHIVE software (Hotan et al. 2004). During the phase-up of the array, data are recorded to measure the relative gain and phase between the vertical and horizontal probes of the receiver per frequency channel. This allows for subsequent polarization calibration to be carried out. Observations of bright quasars are conducted to allow for flux calibration. In the near future these corrections are expected to be applied prior to writing the data to disk. A complete end-to-end description of the observing system is given in Bailes et al. (2019).

In order to achieve the goals of the TPA, careful consideration needs to be given to the total integration time per source per observing session. The integration time per source does not depend (only) on the desired signal-to-noise ratio. Rather, it is also important to gather enough rotations of the pulsar so that pulse-to-pulse fluctuations do not dominate the final profile. There is therefore a trade-off

\footnotetext{
1 Ephemerides were obtained from the pulsar catalogue, available at https://www.atnf.csiro.au/research/pulsar/psrcat/
} 

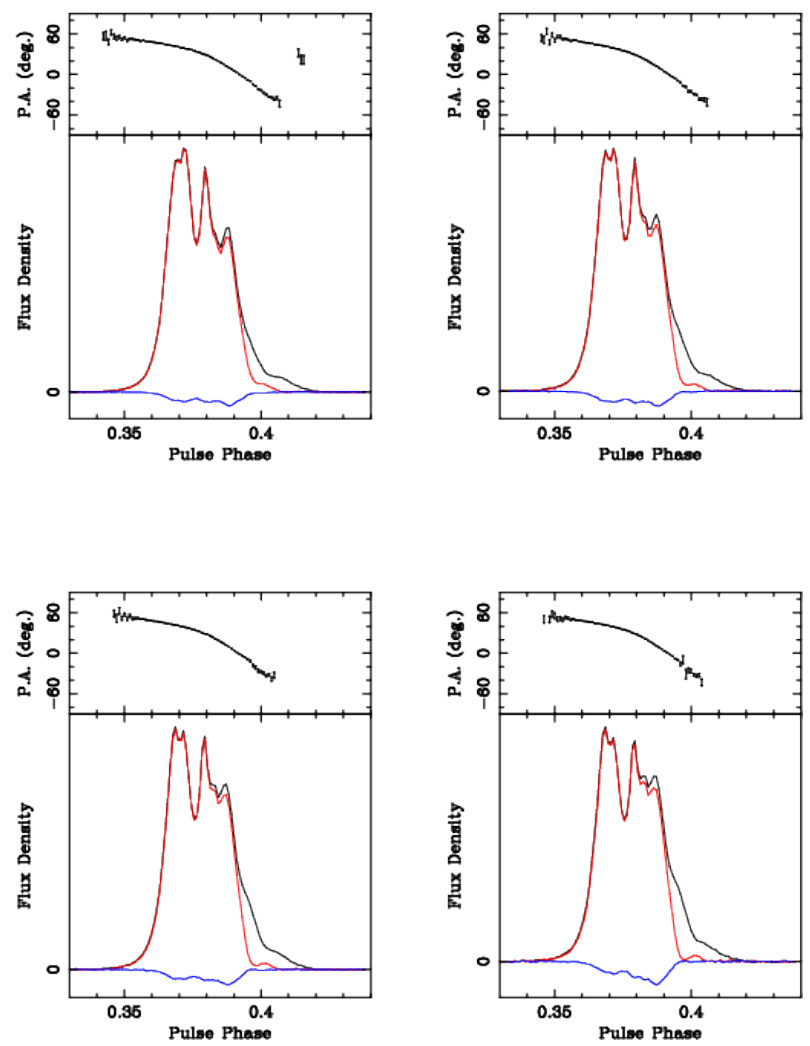

Figure 1. Polarization profiles at centre frequencies of 993 (top left), 1188 (top right), 1381 (bottom left) and 1576 (bottom right) $\mathrm{MHz}$ for PSR J0742-2822. Black denotes total intensity, red linear polarization and blue circular polarization. The top panel shows the position angle of the linear polarization.

between raw sensitivity and observing time and this trade-off is different for the different parts of the project. For the 'single-pulse census' it was decided to observe a minimum of 1024 pulses for all pulsars with a flux density at $1.4 \mathrm{GHz}$ greater than $0.6 \mathrm{mJy}$. For the 'polarimetric census', the integration time is determined via the pulse width, the flux density and the rotation period to ensure both high signal-to-noise ratio and minimum jitter noise. Finally, for the 'repeat monitoring' campaign we require that the telescope can be configured into 2 equal-sized subarrays of 32 antennas. We observe of order 500 pulsars split between the two subarrays with the observing time set to minimise the jitter noise so that profile changes between epochs can be detected at the $10 \%$ level per phase bin. A full description of these trade-offs will be presented in an upcoming publication.

\section{INITIAL RESULTS}

\subsection{Determination of Dispersion Measures}

We can exploit the wide bandwidth of the MeerKAT receiver coupled with the high signal-to-noise ratio of the observations to measure the dispersion measure to high accuracy. The DM is a measure of the total electron number density, $n_{e}$, along the line of sight, $L$, viz

$\mathrm{DM}=\int_{0}^{L} n_{e}(L) d L$

and has the units $\mathrm{cm}^{-3} \mathrm{pc}$.

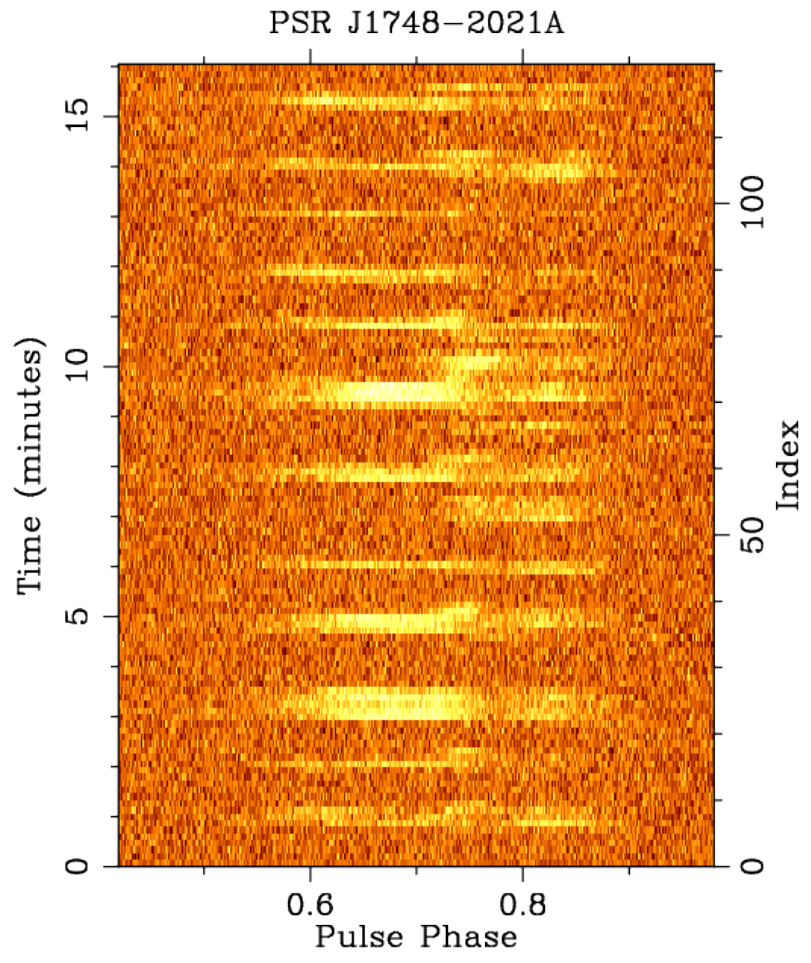

Figure 2. Phase versus time showing nulling in the globular cluster pulsar J1748-2021A. Each horizontal row comprises 8 seconds of data.

Because of the dispersive effects of the interstellar medium, the high frequency radiation arrives at the telescope earlier than the low frequency radiation, and the DM can be derived by a measurement of this time delay viz

$\mathrm{DM}=\frac{t_{2}-t_{1}}{4.149\left(v_{1}^{-2}-v_{2}^{-2}\right)}$

with the observing frequencies, $v_{1}$ and $v_{2}$ in $\mathrm{GHz}$ and the arrival times $t_{1}$ and $t_{2}$ in $\mathrm{ms}$. We therefore determine the DM for each of our pulsars by measuring the delay across the band. In practice this involves the use of the software package TEMPO2 (Hobbs et al. 2006). We first reduce the number of frequency channels by a factor of 32 , and use a single noise-free template to produce a time-of-arrival (ToA) for each channel. TEMPO2 then fits a quadratic function to the ToAs to determine the DM and its uncertainty. Results are given in Table 1 for those pulsars for which the DM differs by more than $3 \mathrm{~cm}^{-3} \mathrm{pc}$ from the previously published value. We are aware that measurements of DM carried out in this standard fashion include a deviation from the true interstellar DM due to profile evolution with frequency and the use of a single template across the band. While previous works (e.g. Pennucci 2019) have introduced techniques to compute frequency-resolved templates for high-precision pulsar timing, these templates only address the frequency dependence of the pulse shape. Frequency-dependent delays are due to a combination of interstellar dispersion and the intrinsic frequency-dependent direction of emission, which is a key aspect to the pulsar emission mechanism and remains an open problem that future TPA papers will address.

We note the curious case of PSR J0905-4536. In the original discovery paper (Manchester et al. 1996) the DM was listed as $189 \pm 9 \mathrm{~cm}^{-3} \mathrm{pc}$, yet in the timing paper of D'Amico et al. (1998) it became $116.8 \pm 0.2 \mathrm{~cm}^{-3} \mathrm{pc}$ which is clearly in error. Bates et al. (2012) spotted this error and computed the DM to be $179.7 \mathrm{~cm}^{-3} \mathrm{pc}$ 


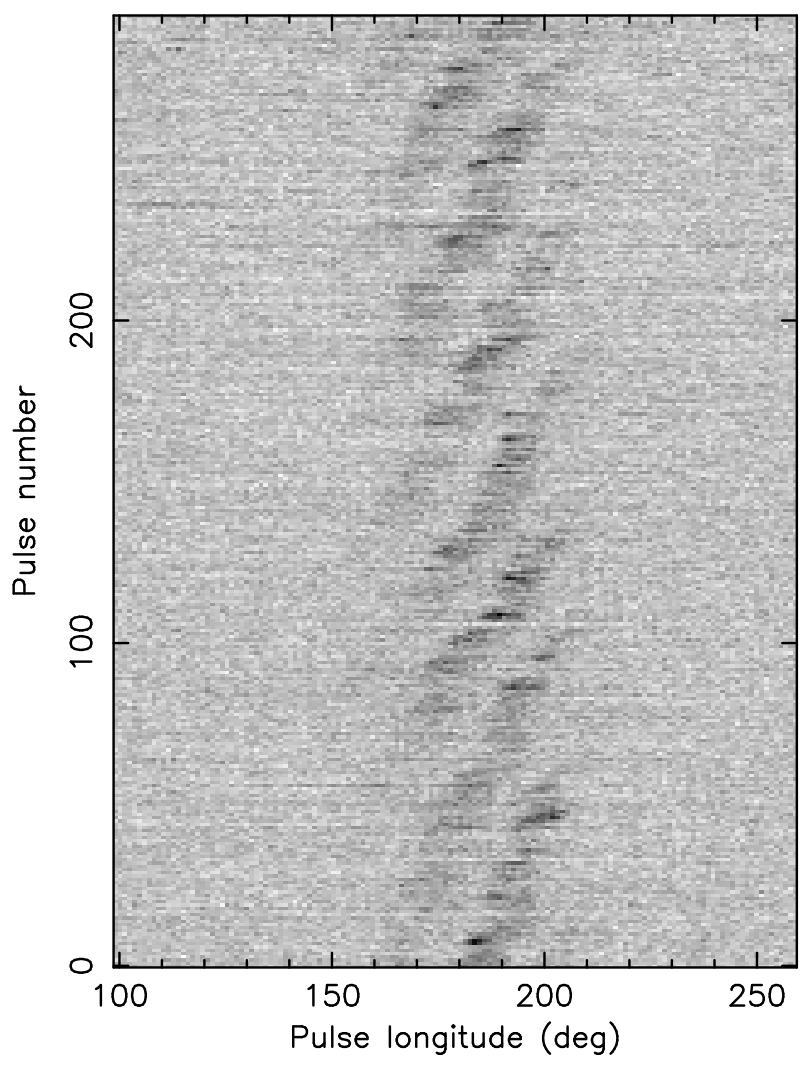

Figure 3. The drifting subpulses of PSR J1750-3503 are revealed in the pulse stack of 295 successive pulses.

(no uncertainty was given). Finally, our observation yields $196.2 \pm$ $0.2 \mathrm{~cm}^{-3} \mathrm{pc}$, consistent (though with much smaller uncertainties) with the original detection! PSRs J1020-6026, J1316-6232, $\mathrm{J} 1527-5552$, J1739+0612, J1814-1744 and J1840-1207 have DMs more than $5 \sigma$ away from their published values. We deem it unlikely that the pulsars' motion through the interstellar medium have caused these changes (see e.g. Petroff et al. 2013). Most likely, the uncertainties were underestimated in the original publications due to the narrow bandwidths employed.

We have observed the bright pulsar PSR J1057-5226 on 6 separate occasions between 2019 February and September. The DM uncertainty for a single 3 minute observation is only $0.003 \mathrm{~cm}^{-3} \mathrm{pc}$, about a factor of 10 better than the equivalent result using the Parkes telescope (Petroff et al. 2013). There is a 2- $\sigma$ significant slope in the measured DMs over the 200 day time span. The small uncertainties imply that multiple observations of bright pulsars over the 5 years of the thousand pulsar array programme should yield detections of DM variations in these slow pulsars, something which has hitherto only been possible for a handful of objects. It may also be possible to exploit the wide bandwidth to measure the 'chromatic' DM effects pointed out by Cordes et al. (2016).

\subsection{Determination of Rotation Measures}

Pulsars are often highly linearly polarized (e.g. Johnston \& Kerr 2018). As the radiation traverses the interstellar medium it suffers Faraday rotation which rotates the position angle of the linearly polarized emission. The rotation measure (RM) is the product of the magnetic field parallel to the line of sight, $\left\langle B_{||}\right\rangle$and the electron

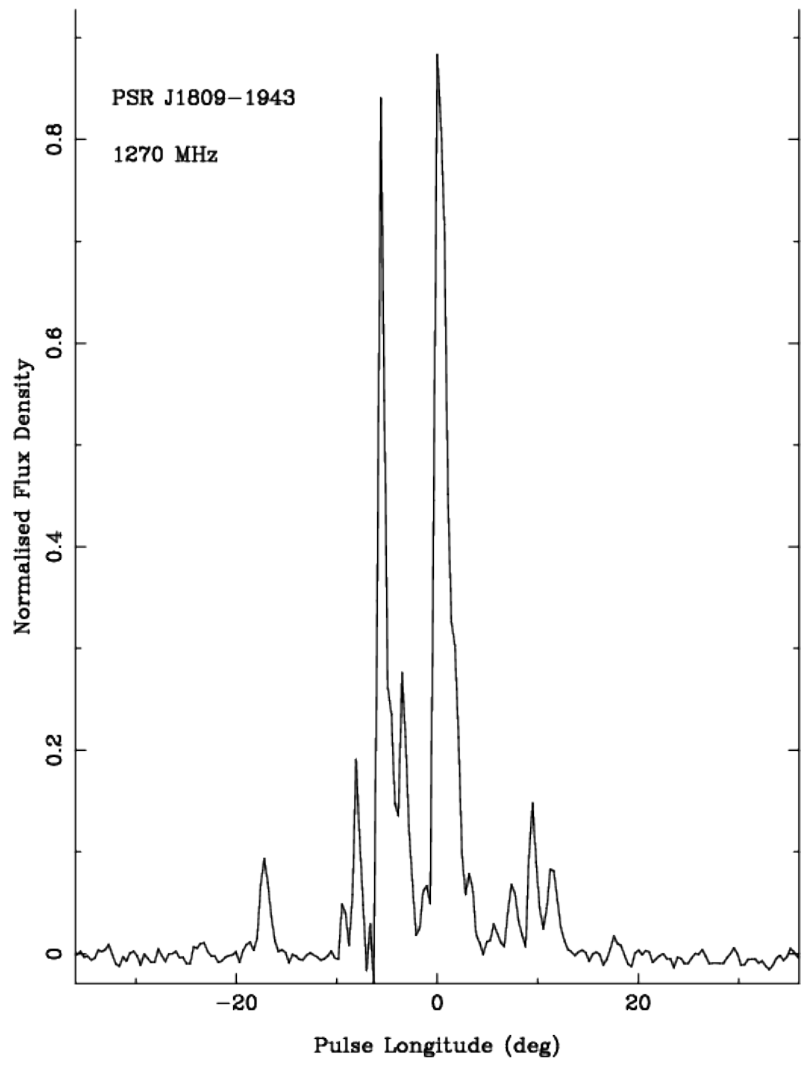

Figure 4. A Stokes I profile of a single pulse of the magnetar XTE J1810-197 (PSR J1809-1943) taken on 2019 March 15

density integrated over the path length to the pulsar:

$\mathrm{RM}=0.81 \int_{0}^{L} n_{e}(L) B_{\|}(L) d L$

With $L$ in pc, $n_{e}$ in $\mathrm{cm}^{-3}$ and $B_{\|}$in $\mu \mathrm{G}$ then the units of RM are $\mathrm{rad} \mathrm{m}^{-2}$. The combination of the RM and the DM yields the average magnetic field strength parallel to the line of sight.

The RM can be determined by measuring the shift in position angle, $\Delta \theta$, at different observing wavelengths, $\lambda_{1}$ and $\lambda_{2}$ :

$\mathrm{RM}=\frac{\Delta \theta}{\lambda_{1}^{2}-\lambda_{1}^{2}}$

In practice we use a combination of methods to compute the RM from the data. One such method is to use trial RMs to de-Faraday the data and then measure the flux density of the linear polarization; the RM at which this peaks is the best RM. This method is similar to the so-called RM synthesis methods used by e.g. Sobey et al. (2019). We also used the method outlined in Noutsos et al. (2008) by measuring the slope to a straight line fit of $\Delta \theta$ versus $\lambda^{2}$. Finally once the RM is known approximately the error bar can be reduced by reducing the number of channels to two and measuring the shift in PA between the two halves of the band. This is the method used by Han et al. (2018).

The RM values for 26 pulsars without previous measurements are given in Table 2 along with the derived $\left\langle B_{\|}\right\rangle$using the pulsar's DM. Uncertainties are $1-\sigma$. Where necessary we also derived an improved DM, as incorrect DM values can result in incorrect RM measurements (e.g. Ilie et al. 2019). These RM values have been corrected for the contribution from the Earth's ionosphere. 

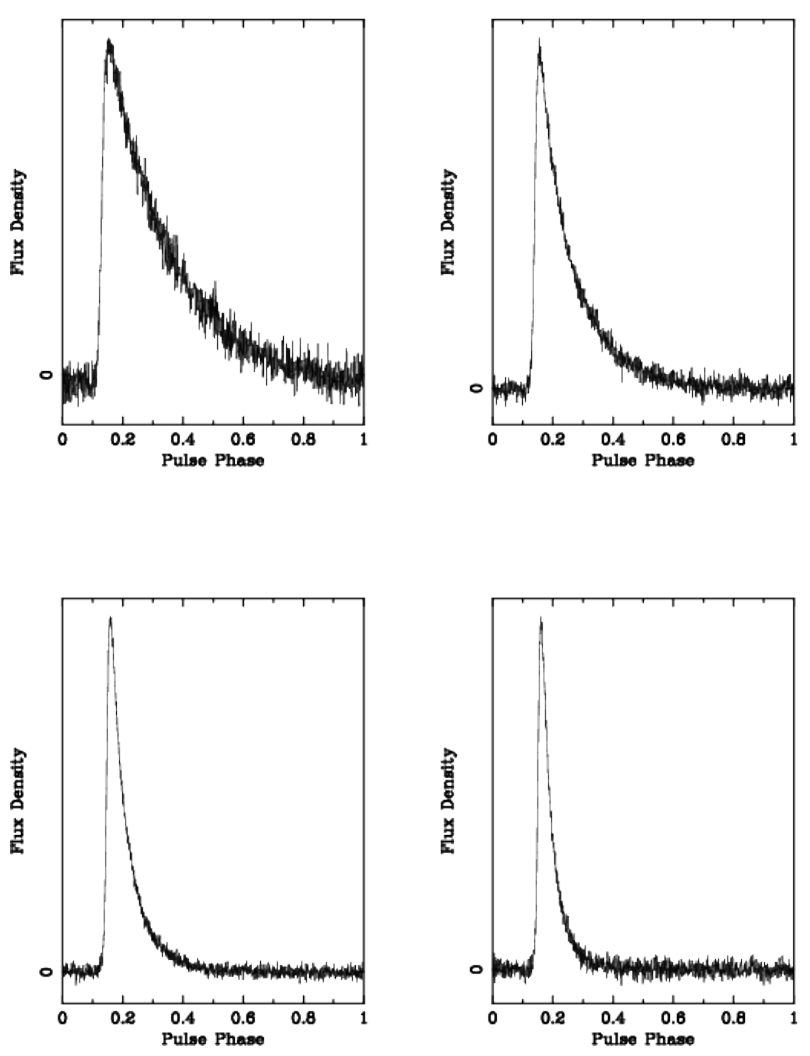

Figure 5. Profiles of PSR J1818-1422 at centre frequencies of 987 (top left), 1183 (top right), 1390 (bottom left) and 1600 (bottom right) $\mathrm{MHz}$ showing the strong dependency of interstellar scattering with frequency.

This contribution was estimated for each pulsar using the publiclyavailable code ionFR ${ }^{2}$ (see Sotomayor-Beltran et al. 2013), with inputs from the International Geomagnetic Reference Field ${ }^{3}$ and International GNSS Service vertical total electron content maps ${ }^{4}$. In all except three cases, the ionospheric contribution to the observed $\mathrm{RM}$ is between -0.2 and $-0.75 \mathrm{rad} \mathrm{m}^{-2}$. For the observations of PSRs J0630-0046, J1104-6103 and J1514-4834 the ionosphere contributes $-1.3 \pm 0.2,-2.0 \pm 0.1$, and $-1.8 \pm 0.1 \mathrm{rad} \mathrm{m}^{-2}$, respectively.

\subsection{Pulsars of interest}

PSR J0045-7319: This pulsar is in the Small Magellanic Cloud and was found to be in orbit about a main sequence B-star (Kaspi et al. 1994; Bell et al. 1995). With a low flux density at $1400 \mathrm{MHz}$, timing at the Parkes telescope has been difficult and observations of the pulsar ceased a decade ago. We carried out two 8 minute observations with the MeerKAT array in 2019 February and May. The pulsar was easily detected with a signal-to-noise ratio of 25 , and is clearly much brighter at the lower frequencies. This promises well for future prospects of determining the spin-orbit coupling in the system (Kaspi et al. 1996).

PSR J0540-6919: This pulsar, with a spin period of only $50 \mathrm{~ms}$, is located in the Large Magellanic Cloud and is known to emit giant pulses (Johnston \& Romani 2003). Observations with the Parkes

\footnotetext{
2 https://github.com/csobey/ionFR/

3 https://www.ngdc.noaa.gov/IAGA/vmod/igrf.html

$4 \mathrm{ftp} / / /$ cddis.nasa.gov/pub/gps/products/ionex/
}

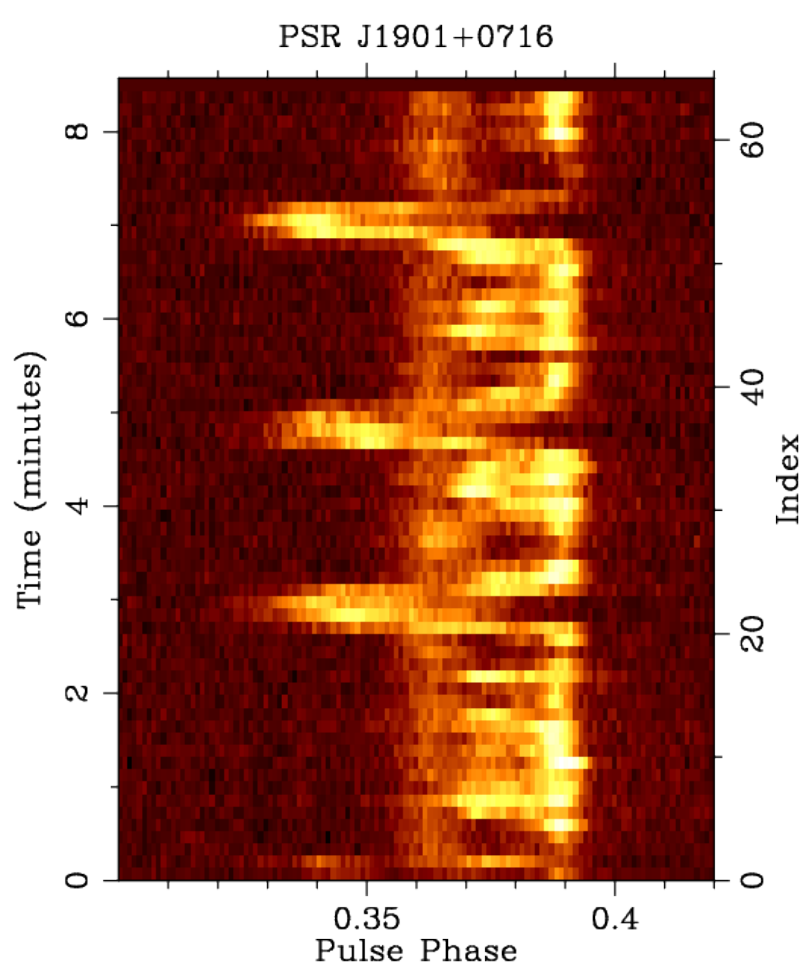

Figure 6. Time versus phase for an 8 minute observation of PSR J1901+0716 showing several transitions between the two states. Each horizontal row represents $8 \mathrm{~s}$ of observing.

telescope detect a giant pulse for every 30 mins of observing time (Johnston et al. 2004), however the sensitivity of MeerKAT means that we detect giant pulses with signal-to-noise ratio $>7$ from the pulsar at a rate greater than 1 per minute. This has allowed us to determine better $\log \mathrm{N}-\log \mathrm{S}$ statistics and for the first time we have measured the polarization properties of the giant pulses. Details will be given in an upcoming paper.

PSR J0742-2822: Polarization calibration of the tied-array remains an on-going challenge at the present time. This pulsar makes a useful test case; it is bright, highly linearly polarized and has a known absolute position angle of the linear polarization (Johnston et al. 2005). Figure 1 shows the polarization profile at 4 different frequencies across the total bandwidth of the receiver. The majority of the features in these profiles are correct, in particular the high fraction of linear polarization with depolarization on the trailing edge of the profile, the negative sign of the circular polarization and the swing of the position angle. Work is in progress to determine the instrumental leakage terms, and to apply the polarization corrections directly at the time of observation without the need to off-line calibration.

PSR J1748-2021A: This pulsar is in the globular cluster NGC 6440 yet has a long spin period of $288 \mathrm{~ms}$. Studies by Lyne et al. (1996) and Freire et al. (2008) did not mention the fact that this pulsar nulls. Our 15 minute observation of the pulsar taken on 2019 July 14 (see Figure 2) shows evidence for nulling and mode switching between the two principal components in the pulse profile. Like all pulsars in the TPA programme with this type of behaviour, further investigation is warranted.

PSR J1750-3503: The pulse phase of the emission of this $684 \mathrm{~ms}$ pulsar changes in a highly periodic fashion from pulse to pulse (see Fig. 3), something not previously reported for this pulsar. The drifting sub-pulse pattern repeats itself every $\sim 50$ rotations of the star, 
which is an exceptionally long timescale compared to other pulsars of this kind (e.g. Rankin 1986; Weltevrede et al. 2006; Basu et al. 2016). This could well be related to to the fact that the characteristic age of this pulsar is very large, $\sim 280 \mathrm{Myr}$. Such a correlation could indeed be expected from a carousel model (Ruderman \& Sutherland 1975). This observation highlights the importance of obtaining a TPA legacy dataset with long enough sequences of single pulses for the brightest pulsars to quantify the pulse-to-pulse variability. XTE J1810-197: Following an X-ray outburst (Gotthelf et al. 2019), this magnetar recently switched back on in the radio and was observed with the Parkes telescope by Dai et al. (2019) and with the Lovell and Effelsberg telescopes by Levin et al. (2019). Figure 4 shows a single pulse from the magnetar in data taken on 2019 March 15 showing the spiky nature of its emission.

PSR J1818-1422: The wide band of the MeerKAT receiver is ideal for study of the scattering imposed by the interstellar medium as the scattering time will vary by a factor of $\sim 20$ from the lowest frequency to the highest. Figure 5 shows the profile of PSR J1818-1422 at 4 different frequencies across the observing band. Details of the functional form of the scattering and the frequency dependence for this and other pulsars from the sample will be presented elsewhere. PSR J1901+0716: This pulsar is known to undergo episodic changes in its emission profile as first identified by Rankin et al. (2006) and studied in detail by Wahl et al. (2016) and Perera et al. (2016). Figure 6 displays 8 minutes of data on the pulsar clearly showing the transition between the states. Long term monitoring of this pulsar will help our understanding of the magnetospheric variability seen at various time-scales, and the link between magnetospheric activity and the spin-down of the star.

\section{SUMMARY}

MeerKAT is delivering high quality and high fidelity pulsar data. We have shown that the polarization properties of the instrument across its $1 \mathrm{GHz}$ of bandwidth are excellent and comparable with single dishes (e.g. Figure 1). The large bandwidth and high sensitivity allow us to measure RMs with high precision even for weak pulsars (e.g. PSR J1722-4400 with a flux density of only $0.2 \mathrm{mJy}$, see Table 2). We have shown that previous DM measurements made from observations of weak pulsars over a narrow bandwidth can be improved with MeerKAT observations (see Table 1). Finally we showed an example of a nulling pulsar (Figure 2) and a drifting pulsar (Figure 3) not previously known. In the near future, the ability to sub-array the 64 antennas, and the installation of the low frequency band receivers (544 to $1088 \mathrm{MHz}$ ) will only enhance the capabilities of this programme. Data from the TPA will be made publicly available once quality control and calibration is complete.

\section{ACKNOWLEDGEMENTS}

The MeerKAT telescope is operated by the South African Radio Astronomy Observatory, which is a facility of the National Research Foundation, an agency of the Department of Science and Innovation. FA and AR acknowledge support through the research grant "iPeska" (P.I. Andrea Possenti) funded under the INAF national call Prin-SKA/CTA approved with the Presidential Decree 70/2016. MB, RS and RMS acknowledge support through ARC grants FL150100148 and CE170100004. AS and JvL acknowledge funding from the Netherlands Organisation for Scientific Research (NWO) under project "CleanMachine" (614.001.301), and from the
ERC under the European Union's Seventh Framework Programme (FP/2007-2013) / ERC Grant Agreement n. 617199. FJ acknowledges funding from the ERC under the European Union's Horizon 2020 research and innovation programme (grant agreement No. 694745). LO acknowledges funding from the UK Science and Technology Facilities Council (STFC) Grant Code ST/R505006/1. AK and BP also acknowledge funding from the STFC consolidated grant to Oxford Astrophysics. Pulsar research at Jodrell Bank Centre for Astrophysics and Jodrell Bank Observatory is also supported by a consolidated grant from STFC.

\section{REFERENCES}

Antoniadis J., et al., 2013, Science, 340, 448

Antonopoulou D., Espinoza C. M., Kuiper L., Andersson N., 2018, MNRAS, 473,1644

Arzamasskiy L. I., Beskin V. S., Pirov K. K., 2017, MNRAS, 466, 2325

Arzoumanian Z., et al., 2018, ApJ, 859, 47

Bailes M., et al., 2019, PASA. Submitted

Barkov M. V., Lyutikov M., Khangulyan D., 2019, MNRAS, 484, 4760

Basu R., Mitra D., Melikidze G. I., Maciesiak K., Skrzypczak A., Szary A., 2016, ApJ, 833, 29

Bates S. D., et al., 2012, MNRAS, 427, 1052

Bell J. F., Bessell M. S., Stappers B. W., Bailes M., Kaspi V. M., 1995, ApJ, 447, L117

Brook P. R., Karastergiou A., Johnston S., Kerr M., Shannon R. M., Roberts S. J., 2016, MNRAS, 456, 1374

Burgay M., et al., 2003, Nature, 426, 531

Camilo F., et al., 2018, ApJ, 856, 180

Cordes J. M., Shannon R. M., Stinebring D. R., 2016, ApJ, 817, 16

D’Amico N., Stappers B. W., Bailes M., Martin C. E., Bell J. F., Lyne A. G., Manchester R. N., 1998, MNRAS, 297, 28

Dai S., et al., 2018, MNRAS, 480, 3584

Dai S., et al., 2019, ApJ, 874, L14

Desvignes G., et al., 2019, Science, 365, 1013

Dyks J., 2019, MNRAS, 488, 2018

Dyks J., Rudak B., 2015, MNRAS, 446, 2505

Espinoza C. M., Lyne A. G., Stappers B. W., Kramer M., 2011, MNRAS, 414, 1679

Freire P. C. C., Ransom S. M., Bégin S., Stairs I. H., Hessels J. W. T., Frey L. H., Camilo F., 2008, ApJ, 675, 670

Freire P. C. C., Kramer M., Wex N., 2012, Classical and Quantum Gravity, 29, 184007

Fuentes J. R., Espinoza C. M., Reisenegger A., Shaw B., Stappers B. W., Lyne A. G., 2017, A\&A, 608, A131

Geyer M., et al., 2017, MNRAS, 470, 2659

Giraud Q., Pétri J., 2019, MNRAS. Submitted., p. arXiv:1910.01555

Gotthelf E. V., et al., 2019, ApJ, 874, L25

Han J. L., Manchester R. N., van Straten W., Demorest P., 2018, ApJSS, 234, 11

Haskell B., Khomenko V., Antonelli M., Antonopoulou D., 2018, MNRAS, 481, L146

Hermsen W., et al., 2013, Science, 339, 436

Hewish A., Bell S. J., Pilkington J. D. H., Scott P. F., Collins R. A., 1968, Nature, 217, 709

Hobbs G. B., Edwards R. T., Manchester R. N., 2006, MNRAS, 369, 655

Hobbs G., et al., 2019, PASA. Submitted, p. arXiv:1911.00656

Hotan A. W., van Straten W., Manchester R. N., 2004, PASA, 21, 302

Ilie C. D., Johnston S., Weltevrede P., 2019, MNRAS, 483, 2778

Jankowski F., et al., 2019, MNRAS, 484, 3691

Johnston S., Karastergiou A., 2017, MNRAS, 467, 3493

Johnston S., Kerr M., 2018, MNRAS, 474, 4629

Johnston S., Romani R. W., 2003, ApJ, 590, L95

Johnston S., Romani R. W., Marshall F. E., Zhang W., 2004, MNRAS, 355, 31 
Johnston S., Hobbs G., Vigeland S., Kramer M., Weisberg J. M., Lyne A. G., 2005, MNRAS, 364, 1397

Jonas J., MeerKAT Team 2016, in Proceedings of MeerKAT Science: On the Pathway to the SKA. 25-27 May. p. 1

Karastergiou A., Johnston S., 2007, MNRAS, 380, 1678

Kaspi V. M., Johnston S., Bell J. F., Manchester R. N., Bailes M., Bessell M., Lyne A. G., D’Amico N., 1994, ApJ, 423, L43

Kaspi V. M., Bailes M., Manchester R. N., Stappers B. W., Bell J. F., 1996, Nature, 381, 584

Kerr M., Hobbs G., Johnston S., Shannon R. M., 2016, MNRAS, 455, 1845

Kerr M., Coles W. A., Ward C. A., Johnston S., Tuntsov A. V., Shannon R. M., 2018, MNRAS, 474, 4637

Klingler N., Kargaltsev O., Pavlov G. G., Ng C. Y., Beniamini P., Volkov I., 2018, ApJ, 861, 5

Kramer M., Lyne A. G., O’Brien J. T., Jordan C. A., Lorimer D. R., 2006a, Science, 312, 549

Kramer M., et al., 2006b, Science, 314, 97

Levin L., et al., 2019, MNRAS, 488, 5251

Lorimer D. R., Bailes M., McLaughlin M. A., Narkevic D. J., Crawford F., 2007, Science, 318, 777

Lyne A. G., Manchester R. N., 1988, MNRAS, 234, 477

Lyne A. G., Manchester R. N., D’Amico N., 1996, ApJ, 460, L41

Lyne A. G., et al., 2004, Science, 303, 1153

Lyne A., Hobbs G., Kramer M., Stairs I., Stappers B., 2010, Science, 329, 408

Lyne A., Graham-Smith F., Weltevrede P., Jordan C., Stappers B., Bassa C., Kramer M., 2013, Science, 342, 598

Manchester R. N., et al., 1996, MNRAS, 279, 1235

McSweeney S. J., Bhat N. D. R., Wright G., Tremblay S. E., Kudale S., 2019, ApJ, 883, 28

Ng C. Y., Romani R. W., 2004, ApJ, 601, 479

Noutsos A., Johnston S., Kramer M., Karastergiou A., 2008, MNRAS, 386, 1881

Olszanski T. E. E., Mitra D., Rankin J. M., 2019, MNRAS, 489, 1543

Oswald L., Karastergiou A., Johnston S., 2019, MNRAS, 489, 310

Özel F., Freire P., 2016, ARA\&A, 54, 401

Palfreyman J., Dickey J. M., Hotan A., Ellingsen S., van Straten W., 2018, Nature, 556, 219

Parthasarathy A., et al., 2019, MNRAS, 489, 3810

Pennucci T. T., 2019, ApJ, 871, 34

Perera B. B. P., Stappers B. W., Weltevrede P., Lyne A. G., Rankin J. M., 2016, MNRAS, 455, 1071

Petroff E., Keith M. J., Johnston S., van Straten W., Shannon R. M., 2013, MNRAS, 435,1610

Philippov A. A., Spitkovsky A., Cerutti B., 2015, ApJ, 801, L19

Pierbattista M., Harding A. K., Grenier I. A., Johnson T. J., Caraveo P. A., Kerr M., Gonthier P. L., 2015, A\&A, 575, A3

Radhakrishnan V., Cooke D. J., 1969, Ap. Lett., 3, 225

Radhakrishnan V., Manchester R. N., 1969, Nature, 222, 228

Rankin J. M., 1986, ApJ, 301, 901

Rankin J. M., 1993, ApJ, 405, 285

Rankin J. M., Rodriguez C., Wright G. A. E., 2006, MNRAS, 370, 673

Rigoselli M., Mereghetti S., Turolla R., Taverna R., Suleimanov V., Potekhin A. Y., 2019, ApJ, 872, 15

Rookyard S. C., Weltevrede P., Johnston S., 2015, MNRAS, 446, 3367

Ruderman M. A., Sutherland P. G., 1975, ApJ, 196, 51

Shannon R. M., Cordes J. M., 2010, ApJ, 725, 1607

Shannon R. M., et al., 2015, Science, 349, 1522

Sobey C., et al., 2019, MNRAS, 484, 3646

Sotomayor-Beltran C., et al., 2013, A\&A, 552, A58

Stairs I. H., et al., 2019, MNRAS, 485, 3230

Szary A., van Leeuwen J., 2017, ApJ, 845, 95

Thornton D., et al., 2013, Science, 341, 53

Wahl H. M., Orfeo D. J., Rankin J. M., Weisberg J. M., 2016, MNRAS, 461, 3740

Walker M. A., Koopmans L. V. E., Stinebring D. R., van Straten W., 2008, MNRAS, 388, 1214

Walker M. A., Demorest P. B., van Straten W., 2013, ApJ, 779, 99
Weltevrede P., Edwards R. T., Stappers B. W., 2006, A\&A, 445, 243

Weltevrede P., et al., 2010, PASA, 27, 64

Wright G., Weltevrede P., 2017, MNRAS, 464, 2597

Yao J. M., Manchester R. N., Wang N., 2017, ApJ, 835, 29

van Straten W., Bailes M., 2011, PASA, 28, 1

This paper has been typeset from a $\mathrm{TE}_{\mathrm{E}} \mathrm{X} / \mathrm{L} \mathrm{T} \mathrm{E}$ file prepared by the author. 